\title{
Guía de Valorización de la Idoneidad Didáctica de Procesos de Instrucción en Didáctica de la Estadística
}

\author{
Valuation Guide of Didactic Suitability of Instructional Processes in \\ Statistic Didactics
}

\author{
Felipe Ruz* \\ ORCID iD 0000-0003-4050-728X \\ Elena Molina-Portillo** \\ ORCID iD 0000-0002-9955-3080 \\ José M. Contreras García ${ }^{* * *}$ \\ ORCID iD 0000-0001-6821-0563
}

\begin{abstract}
Resumen
Reconociendo el importante rol que cumplen los docentes en el proceso de enseñanza y aprendizaje de la estadística y probabilidad, en este artículo abordamos el problema de la valoración de planes de formación de profesores de matemática en didáctica de la estadística. Para ello, se describe el proceso de construcción de una guía que valorice su idoneidad, según una colección de indicadores inferidos por medio de la técnica de análisis de contenido de documentos, de consenso internacional, que rigen u orientan esta etapa formativa. Como consecuencia, la guía resultante se organiza en dos dimensiones de análisis, una que nos permita caracterizar el conocimiento didáctico-matemático del futuro profesor según las expectativas de aprendizaje institucionales de sus estudiantes (faceta epistémica), y una segunda que considera las responsabilidades del formador con los docentes en formación (facetas restantes). Esperamos que este instrumento sea un insumo valioso tanto para formadores como para quienes tengan la tarea de diseñar o evaluar planes formativos para futuros docentes.
\end{abstract}

Palabras clave: Formación de Profesores. Idoneidad Didáctica. Guía de valoración. Planes de Formación. Didáctica de la Estadística.

\begin{abstract}
In order to recognize the important role of teachers in the discipline's teaching and learning processes, in this article, we confront the problem of valuating the teaching planning in statistics didactics. For this, we describe

\footnotetext{
* Magíster por la Pontificia Universidad Católica de Valparaíso (PUCV). Doctorando en la Universidad de Granada (UGR), Granada, España. Dirección postal: Departamento de Didáctica de la matemática, Facultad de Ciencias de la Educación, Campus de Cartuja, s/n., Granada, España, C.P: 18071. E-mail: felipe.ruz.angel@gmail.com.

${ }^{* *}$ Doctora por Universidad de Granada (UGR). Profesora sustituta interina en la Universidad de Granada (UGR), Granada, España. Dirección postal: Departamento de Didáctica de la matemática, Facultad de Ciencias de la Educación, Campus de Cartuja, s/n. Granada, España, C.P: 18071. E-mail: elemo@ugr.es.

*** Doctor por la Universidad de Granada (UGR). Profesor contratado doctor en la Universidad de Granada (UGR), Granada, España. Dirección postal: Departamento de Didáctica de la matemática, Facultad de Ciencias de la Educación, Campus de Cartuja, s/n., Granada, España, C.P: 18071. E-mail: jmcontreras@ugr.es.
} 
the construction process of a guide that values its suitability according to a collection of indicators inferred through the content analysis technique of international consensus documents that guide this formative stage. As a consequence, the resulting guide is organized into two analysis dimensions, one that allows us to characterize the didactic-mathematical knowledge of the future teacher according to the institutional learning expectations of its students (epistemic facet) and the second that considers the trainer responsibilities with teachers in training (remaining facets). We hope that this instrument is a valuable input for both, trainers and those who have the task of designing or evaluating teaching planning for future teachers.

Keywords: Teachers' Education. Didactical Suitability. Valuation Guide. Teaching Planning. Didactics of Statistic.

\section{Introducción}

En la actualidad, la estadística es considerada como parte de la herencia cultural necesaria para todo ciudadano, siendo valorada por diversos agentes educativos y políticos como un componente básico para desenvolverse eficazmente en la sociedad de la información. En ella, debemos ser conscientes de las tendencias y fenómenos de importancia social y personal, ser capaces de tomar decisiones en situaciones de incertidumbre y participar de manera informada en el debate público y político (GAL, 2002).

En este sentido, en un entorno donde diariamente nos convertimos en consumidores de datos (ciudadanos receptores de información estadística), debido a que los datos son utilizados, cada vez más, para añadir credibilidad a la información presente en los medios de comunicación, la toma de decisiones políticas y económicas, ser personas estadísticamente educadas se reconoce como una competencia necesaria para mejorar la calidad de vida, dado que con ella se espera desarrollar nuestra capacidad de monitorear y promover la justicia social (BEN-ZVI; MAKAR, 2016).

En los últimos años, ha aparecido una corriente reformista en torno a la educación estadística en la escuela, promoviendo su enseñanza, dentro del currículo de matemática, desde los niveles iniciales de primaria, con ideas básicas de la disciplina, hasta terminar la trayectoria escolar con elementos de inferencia estadística (p. e. Estados Unidos: NCTM, 2000; FRANKLIN et al., 2007; España: MECD, 2015; Chile: MINEDUC, 2009).

De esta forma, el éxito de los nuevos programas u orientaciones curriculares depende, en gran medida, de la formación de quienes tienen la tarea de su implementación, ya que los profesores de matemática "son la fuerza impulsora de la reforma en educación estadística" (BEN-ZVI; MAKAR, 2016, p. 6), por ser los responsables de adaptar e interpretar los nuevos requerimientos según las características y condiciones institucionales, de sus estudiantes y del entorno educativo. Por tanto, identificando que la clave para prosperar en la educación estadística comienza por los profesores en sí mismos, en este trabajo analizamos su formación 
inicial, centrando nuestra atención en los programas de estudio que regulan dicho proceso, de manera que podamos valorizar su grado de idoneidad respecto a las exigencias actuales declaradas en diversos documentos de consenso internacional y local para Chile.

El diseño curricular que norma la formación de profesores y regula sus procesos de instrucción, se lleva a cabo, usualmente, por distintos agentes educativos intervinientes. Por ejemplo, en la realidad chilena la formación de profesores está normada por el Ministerio de Educación a través de la conducción técnica del Centro de Perfeccionamiento, Experimentación e Investigaciones Pedagógicas (MINEDUC; CPEIP, 2012) y la colaboración de diversos centros de investigación específicos de esta materia. Luego, son las instituciones de educación superior que ofrecen carreras de pedagogía quienes a través de sus distintas entidades (institutos, escuelas, departamentos o seminarios) formulan programas de estudio específicos para cada centro y, por último, el formador a cargo de impartir docencia es el responsable de elaborar las planificaciones de aula específicas, que idealmente deben ser complementadas con resultados de la investigación educativa y didáctica en general.

De esta forma, aunque el formador maneje y domine los principios disciplinares, pedagógicos y didácticos orientadores del diseño curricular, su aplicación a temas específicos "deja demasiados grados de libertad, lo que torna insegura y excesivamente compleja la acción del docente" (GODINO et al., 2013, p. 48). Lo que aplicado al campo de la enseñanza de la estadística y la probabilidad podría justificar lo mencionado por Batanero, Burrill y Reading (2011), quienes aseguran que muchos docentes consideran que no están bien preparados para enseñar estadística ni para afrontar las dificultades que este proceso implica.

Por lo tanto, con la intención de aportar herramientas que apoyen la mejora de la formación estadística de futuros profesores de matemáticas, en este artículo se presenta el proceso de construcción de una Guía de Valoración de la Idoneidad Didáctica de procesos de Instrucción en Didáctica de la Estadística (GVID-IDE), conformada por un serie de indicadores empíricos que permitan evaluar programas de formación de manera operativa y apoyen la reflexión sistémica sobre la enseñanza y aprendizaje de la didáctica de la estadística, según la gama de facetas de análisis que son parte de la Teoría de Idoneidad Didáctica (GODINO, 2013; BREDA; FONT; LIMA, 2015).

A continuación, en la sección 2 se describe el problema y marco teórico que respaldan el enfoque adoptado en esta investigación, destacando la noción de Idoneidad Didáctica, sus facetas y componentes. En la sección 3 se detalla y ejemplifica la metodología empleada en el proceso de construcción y validación de la GVID-IDE, tratando de destacar las etapas claves, de manera que puedan ser de utilidad para investigadores interesados en esta área y que 
puedan complementar nuestros resultados con el análisis de los procesos de formación en otras áreas específicas de las matemáticas (álgebra, geometría, números). Posteriormente, en la sección 4 se presenta la totalidad de indicadores de idoneidad que constituyen la GVIDIDE, destacando la manera de interpretarlos según la faceta o componente a la que pertenezcan. Por último, el artículo concluye con algunas reflexiones finales y proyecciones de la investigación reportada.

\section{Problema y marco teórico}

En este trabajo afrontamos el problema de evaluar planes de formación de profesores de matemática en el ámbito de didáctica de la estadística desde la perspectiva aportada por el Enfoque Ontosemiótico del Conocimiento y la Instrucción Matemáticos - EOS (GODINO; BATANERO; FONT, 2007). El EOS presenta como un sistema teórico inclusivo, abierto y dinámico, que desde sus inicios se ha planteado como problemática de investigación y reflexión la comparación y articulación de marcos teóricos, aspirando a incluir en él las nociones teóricas y metodológicas "necesarias y suficientes" (GODINO, 2012, p. 56) para investigar la complejidad de los procesos de enseñanza y aprendizaje de las matemáticas y la estadística.

Hasta la fecha, la evolución de este sistema teórico ha concluido en cinco grupos de herramientas que permiten analizar aspectos complementarios de los procesos de enseñanza y aprendizaje de las matemáticas, entre los que tomamos la Teoría de Idoneidad Didáctica (GODINO, 2013) para abordar la problemática del diseño y valoración de planes formativos en didáctica de la estadística para profesores de matemática de los niveles 7 al 12 (estudiantes de doce a dieciocho años de edad), cuyo desarrollo y aplicación fue tratado en Godino et al. (2013) para el caso de un plan de formación en didáctica de la matemática para profesores de educación primaria.

Los elementos en los que se fundamenta la Teoría de Idoneidad Didáctica han sido introducidos de forma gradual al EOS, a partir de los trabajos de Godino, Contreras y Font (2006) y Godino et al. (2006), como herramientas de una didáctica normativa, orientada a la intervención efectiva en el aula. La noción de idoneidad didáctica es entendida como un criterio sistémico de pertinencia de un proceso de instrucción, respecto al grado de adaptación entre dos tipos de significados, los personales logrados por los estudiantes y los pretendidos o implementados según la institución de referencia. De esta forma, la idoneidad de un proceso de instrucción (pretendido/programado o implementado) se define como la articulación 
coherente y sistémica de seis facetas y sus posibles interacciones entre sí (GODINO, 2013):

- Idoneidad epistémica: Referida al grado de representatividad de los significados institucionales pretendidos o implementados respecto de un significado de referencia.

- Idoneidad cognitiva: expresa el grado en que los significados pretendidos o implementados están en la zona de desarrollo potencial o son próximos a los significados personales de los estudiantes.

- Idoneidad afectiva: representa el grado de implicación (interés, motivación etc.) de los estudiantes con el proceso de estudio, que puede verse influenciado tanto por factores institucionales como personales.

- Idoneidad interaccional: refleja el grado en que las configuraciones y trayectorias didácticas, que son parte del proceso de enseñanza y aprendizaje, identifiquen y resuelvan conflictos semióticos potenciales.

- Idoneidad mediacional: expresa el grado de disponibilidad y adecuación de los recursos materiales y temporales necesarios para el desarrollo del proceso de enseñanza y aprendizaje.

- Idoneidad ecológica: representa el grado en que el proceso de estudio de ajusta al proyecto educativo institucional y a los condicionamiento del entorno en que se desarrolla.

A su vez, reconociendo la complejidad de evaluar la idoneidad de un proceso de instrucción, debido al gran de número de aspectos que intervienen en él, Godino (2013) identifica una serie de componentes que articulan y sistematizan el análisis de cada una de las facetas mencionadas anteriormente. De esta forma, debido a que tanto las facetas como los componentes de idoneidad didáctica no son observables directamente, es necesario inferirlos a partir de indicadores empíricos, entendidos como heurísticas que consideran las restricciones propias del contexto y de la faceta correspondiente (BELTRÁN-PELLICER; GODINO, 2017).

Godino (2013) propone una Guía para la Valoración de la Idoneidad Didáctica de procesos de Instrucción Matemática (GVID-IM), compuesta por una colección de indicadores que sintetizan los elementos y relaciones que intervienen en un proceso de instrucción matemática de cualquier nivel escolar. Sin embargo, puesto que nuestro interés es valorar planes de formación en didáctica de la estadística, consideramos los resultados de Godino et al. (2013) que representan la evolución de la GVID-IM para convertirla en un instrumento de reflexión sobre procesos de instrucción acerca de los conocimiento didáctico-matemáticos de 
profesores en formación, denominada Guía de Valoración de la Idoneidad Didáctica de procesos de Instrucción en Didáctica de la Matemática (GVID-IDM).

La GVID-IDM se construye sobre la base de los trabajos realizados en Godino (2009) (cuya perspectiva ampliada se desarrolla en PINO-FAN; GODINO, 2015), donde se integran, organizan y extienden tres referentes teóricos sobre conocimiento y desarrollo profesional del profesor de matemáticas que han tenido gran aceptación en la comunidad educativa: (1) el Modelo de Conocimiento Pedagógico del Contenido - PCK (SHULMAN, 1987), entendido como el conocimiento necesario para la enseñanza de un cierto tema, que hace interactuar el conocimiento del contenido y el currículo; (2) el Modelo de Conocimiento Matemático para la Enseñanza - MKT (BALL, 2000; HILL; BALL; SHILLING, 2008), entendido como el conocimiento disciplinar necesario para que el profesor pueda conducir tanto la instrucción como el aprendizaje de sus estudiantes; y (3) la Teoría de desempeño experto en enseñanza de las matemáticas (SCHOENFELD; KILPATRICK, 2008), referida a los conocimientos y competencias profesionales del profesor de matemáticas para que su enseñanza pueda considerarse de calidad.

Con todo ello, Godino (2009) fusiona los tres referentes mencionados y aplica la diversidad de herramientas disponibles en el EOS, proponiendo un nuevo modelo de lo que denomina Conocimiento Didáctico-Matemático (CDM) del profesor, cuya aplicabilidad al campo de la estadística se expone en Godino et al. (2011). De esta forma, Godino et al. (2013) adoptan el modelo CDM para garantizar que la GVID-IDM condense, a través de indicadores, el conocimiento necesario por el profesor de matemática para llevar a cabo su labor.

Sobre los cimientos teóricos declarados anteriormente y los componentes de la GVIDIDM que se resumen en el Cuadro 1, se diseña una Guía de Valoración de la Idoneidad Didáctica de procesos de Instrucción en Didáctica de la Estadística (GVID-IDE). De esta forma, para la construcción de un instrumento que permita evaluar un proceso de instrucción (pretendido/programado o implementado) de formación de profesores en didáctica de la estadística, es necesario distinguir dos dimensiones desde las cuales analizarlo: una que nos permita caracterizar la labor del futuro profesor según las expectativas de aprendizaje institucionales de sus estudiantes, y una segunda que considere las tareas del formador con los docentes en formación (ambos aspectos son representados por cada columna del Cuadro 1).

\begin{tabular}{|l|}
\hline \multicolumn{1}{|c|}{ FACETA EPISTÉMICA } \\
Contenido Didáctico-Matemático, entendido desde el \\
punto de vista institucional
\end{tabular}

\begin{tabular}{l} 
OTRAS FACETAS IMPLICADAS \\
EN LA FORMACIÓN EN DIDÁCTICA DE LA \\
MATEMÁTICA \\
\hline FACETA COGNITIVA: \\
$\begin{array}{l}\text { Aprendizaje del contenido didáctico-matemático } \\
\text { por los profesores }\end{array}$
\end{tabular}




\begin{tabular}{|l|}
\hline \multicolumn{1}{|c|}{ Contenido cognitivo: } \\
Conocimientos previos, adaptaciones curriculares, \\
aprendizaje del contenido matemático por parte de \\
los alumnos
\end{tabular}

\begin{tabular}{|l|}
\hline \multicolumn{2}{|c|}{ FACETAAFECTIVA: } \\
$\begin{array}{l}\text { Creencias, valores, intereses, actitudes, } \\
\text { emociones de los profesores hacia el aprendizaje } \\
\text { del contenido didáctico-matemático }\end{array}$ \\
\hline \multicolumn{1}{|c|}{ FACETA INTERACCIONAL: } \\
Modos de interacción y discurso en el proceso de \\
formación de profesores \\
\hline \multicolumn{1}{|c|}{ FACETA MEDIACIONAL: } \\
$\begin{array}{l}\text { Uso de recursos tecnológicos en el proceso de } \\
\text { formación de profesores }\end{array}$ \\
\hline $\begin{array}{l}\text { FACETA ECOLÓGICA: } \\
\text { Currículo, innovación didáctica en formación de } \\
\text { profesores, conexiones interdisciplinares }\end{array}$ \\
\hline
\end{tabular}

Cuadro 1 - Componentes de la GVID-IDM

Fuente: Godino et al. (2013, p. 54)

Organizamos la primera de dichas dimensiones dentro de la Faceta Epistémica del instrumento GVID-IDE, referida a los conocimientos institucionales sobre la enseñanza y aprendizaje de la estadística, que considera diversos contenidos didáctico-estadísticos (disciplinar, cognitivo, afectivo, interaccional, mediacional y ecológico) que intervienen en la labor profesional del profesor en formación respecto a sus futuros estudiantes. La segunda dimensión es contemplada en las Facetas restantes que involucran al formador con sus estudiantes (profesores en formación) en términos: (1) Cognitivos, referido al logro efectivo de las expectativas de aprendizaje en didáctica de la estadística; (2) Afectivos, actitudes y motivaciones de los futuros profesores hacia la enseñanza de la estadística; Interaccionales, competencias comunicativas del profesor en formación con su formador y pares; (4) Mediacionales, uso de recursos disponibles para el planteamiento de situaciones vinculadas con la práctica de la enseñanza y (5) Ecológico, que contemple el conocimiento del formador en cuanto al currículo respectivo, el uso de nuevas tecnologías basadas en la investigación y la conexiones con otras áreas disciplinares.

A continuación, articulamos los aspectos teóricos mencionados anteriormente y exponemos un desglose metodológico respecto al proceso de construcción de la GVID-IDE.

\section{Metodología}

Esta investigación se cataloga dentro del enfoque cualitativo y se considera de tipo exploratoria descriptiva (HERNÁNDEZ; FERNÁNDEZ; BAPTISTA, 2014) donde la técnica empleada para la recogida de información es el análisis de contenido (KRIPPENDORFF, 1990). Esta técnica, considera que lo útil del fragmento de mensaje a analizar es la presencia o 
ausencia de una característica del contenido y es utilizada para identificar, clasificar y comparar normas organizadas en categorías, según las distintas facetas de idoneidad didáctica descritas en la sección 2, contenidas en documentos curriculares de consenso internacional y específicos para la realidad chilena, que rigen la formación de profesores en el eje de estadística y probabilidades. La elección de estos documentos se realiza por medio de un muestreo intencional de carácter teórico, fundamentado, principalmente, en el objetivo que se persigue con este estudio y la pertinencia de ellos respecto a toda la colección de documentos curriculares disponibles en la literatura que regulan u orientan la formación inicial docente en esta área.

El primero de estos documentos corresponde al informe Statistical Education of Teachers - SET (FRANKLIN et al., 2015), desarrollado por la Asociación Americana de Estadística (ASA), donde se describe el contenido y la comprensión conceptual que los profesores necesitan saber para ayudar a sus estudiantes a desarrollar habilidades de razonamiento estadístico. Este reporte se destina a todos los que participan en la educación estadística de los profesores, tanto en su proceso de preparación inicial como en la formación continua.

En cuanto al contenido analizado en este documento, seleccionamos las orientaciones propuestas para la formación de profesores de middle (niveles 6 al 8) y high school (niveles 9 al 12), omitiendo lo referido a los niveles inferiores, pues el alcance de este estudio está orientado a la valoración de planes formativos de futuros profesores que impartirán docencia escolar entre los niveles seleccionados.

El segundo documento, denominado Estándares orientadores para carreras de pedagogía en educación media (MINEDUC; CPEIP, 2012), que tiene por finalidad orientar a las instituciones formadoras de profesores chilenos, respecto a aquellos conocimientos y habilidades que debe demostrar un futuro profesor de educación media (niveles 7-12) para enseñar la disciplina en los seis grados que comprende este nivel de escolaridad. Este informe se organiza en torno a dos aspectos: (1) los estándares pedagógicos, que corresponden a las competencias necesarias para el desarrollo del proceso de enseñanza, considerando aspectos como: la dimensión moral de la profesión, gestión de la clase, interacción con los estudiantes y promover un ambiente adecuado para el aprendizaje y (2) los estándares disciplinarios para la enseñanza, que sugieren los conocimientos y habilidades disciplinares que los futuros profesores deben demostrar, incluido el cómo se enseña, considerando atributos como: la comprensión sobre cómo aprenden los estudiantes, la capacidad para diseñar, planificar e implementar experiencias de aprendizaje y su evaluación. 
Entre ambas colecciones de estándares, fueron analizados completamente los primeros, pero de los segundos observamos los estándares de matemática, particularmente los que conforman el eje de datos y azar que son nuestro foco de interés (del estándar 17 al 21 con sus respectivas descripciones e indicadores).

Otro documento, considerado inicialmente, corresponde a la última actualización del informe universitario Guidelines for Assessment and Instruction in Statistics Education (GAISE, 2016) presentado por primera vez el año 2005 por la ASA, donde se sugiere una dirección para orientar los cursos introductorios de estadística en el nivel superior. Sin embargo, compartimos lo mencionado en Franklin et al. (2015) respecto a que tanto este informe como el Common Core State Standards for Mathematics (CCSSM) (NGACBP; CCSSO, 2011) y Principles and Standards for School Mathematics (NCTM, 2000) incluyen orientaciones que "no son adecuadas para preparar a los profesores para enseñar estadística" (FRANKLIN et al., 2015, p. 24), ya que dependiendo del interés profesional a quienes vayan dirigidas, usualmente prestan poca atención al ciclo de investigación empírica, proporcionan un enfoque axiomático a la probabilidad y la inferencia se aborda como una colección de procedimientos de memoria, por lo que fueron descartados.

Los reportes Franklin et al. (2015) y MINEDUC-CPEIP (2012) en conjunto, contienen normas que orientan el quehacer formativo de futuros profesores de matemática en lo que respecta a la estadística y las probabilidades, por tanto, representan una parte importante del conocimiento profesional que un profesor debe manejar. A partir de dichas normas se infieren indicadores clasificados según las facetas de idoneidad, proceso que se detalla y ejemplifica en lo que sigue.

En una primera fase, se identificaron y clasificaron Unidades de Análisis (UA) según las distintas facetas de idoneidad que, posteriormente, fueron sometidas a valoración por juicio de expertos para garantizar la validez del contenido resultante en cada categoría y asegurar su correcta clasificación. Este procedimiento involucró a dos académicos con una amplia trayectoria de investigación en el campo de la didáctica de la matemática y estadística, sumado a un total dominio del marco teórico y metodología seguida. De esta forma, los resultados de dicha valoración permitieron una nueva revisión de la clasificación propuesta, reubicando un número reducido de UA que produjeron discrepancia.

Continuando con la investigación, fue necesario comparar y reducir las UA resultantes de la etapa anterior con la intención de evitar reiteraciones. Este procedimiento consiste en identificar y etiquetar las UA que puedan estar contenidas en otra, o que no aporten información nueva, con la expresión contenida en acompañada de la letra (a, b, c...) de la UA 
correspondiente para dejar representado su contenido en una única Unidad de Análisis final. En el Cuadro 2, se ejemplifica el procedimiento explicado, donde las letras no siguen un orden correlativo ya que se mantiene su clasificación original y se presentan aquellas que contienen o están contenidas en otras.

\begin{tabular}{|c|c|}
\hline COMPONENTES & UNIDADES DE ANÁLISIS \\
\hline 2.3. Aprendizaje & $\begin{array}{l}\text { d. "razonan inductivamente los datos, haciendo inferencias que toman en } \\
\text { cuenta el contexto de donde surgieron los datos" (FRANKLIN et al., 2015, } \\
\text { p. 11) contenida en g. } \\
\text { e. "entiende que los modelos estadísticos se juzgan según su utilidad y que } \\
\text { describan razonablemente los datos" (FRANKLIN et al., 2015, p. 11). } \\
\text { g. "Razonan en presencia de variabilidad y anticipan, reconocen, explican y } \\
\text { permiten la variabilidad en los datos, ya que se relaciona con un contexto } \\
\text { particular" (FRANKLIN et al., 2015, p. 10). } \\
\text { h. "comprender el rol de la variabilidad en la resolución de problemas } \\
\text { estadísticos" (FRANKLIN et al., 2015, p. 21) contenida en g. } \\
\text { i. "Entender que la distribución de los datos describe la variabilidad presente } \\
\text { en ellos" (FRANKLIN et al., 2015, p. 22) contenida en g. } \\
\text { jj. "Conoce la aproximación de la distribución binomial por la distribución } \\
\text { Poisson y la usa para estimar probabilidades" (MINEDUC; CPEIP, 2012, p. } \\
\text { 128) contenida en e. } \\
\text { kk. "Conoce propiedades básicas de la distribución exponencial y resuelve } \\
\text { problemas que la involucren" (MINEDUC; CPEIP, 2012, p. 130) contenida } \\
\text { en e. } \\
\text { ll. "Comprende la Ley de los Grandes Números y la aplica para resolver } \\
\text { problemas" (MINEDUC; CPEIP, 2012, p. 130) contenida en e. }\end{array}$ \\
\hline
\end{tabular}

Cuadro 2 - Ejemplo del proceso de comparación y reducción de la UA Fuente: Resultados de la investigación

Tras comparar y reducir las distintas UA se procede a inferir indicadores de Idoneidad Didáctica a partir de los fragmentos resultantes, considerando que: (1) dos o más UA pueden dar origen a un único indicador, y (2) una misma UA puede dar origen a uno o más indicadores. De esta forma, se obtiene una propuesta de indicadores para valorar procesos de formación en didáctica de la estadística para futuros profesores de matemática, que se ejemplifica en el Cuadro 3. En este punto es importante mencionar que, con la intención de proponer una guía que pueda utilizarse tanto para valorar planes como acciones formativas, se han redactado los indicadores en un sentido global reemplazando expresiones como resuelven por promueven o incluyen, ya que la primera sólo podría aplicarse a un proceso de estudio implementado y no en la etapa de diseño.

\begin{tabular}{|c|c|}
\hline 14 & \\
\hline $\begin{array}{l}\text { d. "entiende que los modelos estadísticos se juzgan según su } \\
\text { utilidad y que describan razonablemente los datos" } \\
\text { (FRANKLIN et al., 2015, p. 11). } \\
\text { t. "Es capaz de conceptualizar, analizar, sintetizar, argumentar, } \\
\text { interpretar, evaluar, inferir y explicar ideas o temas en forma } \\
\text { oral o escrita" (MINEDUC; CPEIP, 2012, p. 45) } \\
\text { u. "Comprende la importancia que tiene la forma de presentar } \\
\text { los datos de manera gráfica y conoce las precauciones que } \\
\text { deben considerarse al utilizar estas herramientas" } \\
\text { (MINEDUC; CPEIP, 2012, p. 124). }\end{array}$ & $\begin{array}{l}\text { - Se promueve el correcto } \\
\text { entendimiento de los modelos } \\
\text { estadísticos, que se juzgan según } \\
\text { su utilidad y descripción de los } \\
\text { datos (UAd, u) } \\
\text { - Se incluye la preparación para } \\
\text { conducir el aprendizaje de los } \\
\text { tópicos de análisis de datos, } \\
\text { probabilidades e inferencia } \\
\text { estadística presentes en el }\end{array}$ \\
\hline
\end{tabular}


w. "Está preparado para conducir el aprendizaje de las variables currículo escolar (UA t, w, x) aleatorias discretas" (MINEDUC; CPEIP, 2012, p. 128).

x. "Está preparado para conducir el aprendizaje de inferencia estadística" (MINEDUC; CPEIP, 2012, p. 132).

\section{Cuadro 3 - Ejemplo del proceso de inferencia de indicadores de idoneidad \\ Fuente: Resultados de la investigación}

Finalmente, del proceso descrito anteriormente, hemos obtenido una primera versión de la GVID-IDE que, posteriormente, fue sometida a confrontación con la literatura existente acerca del análisis de la idoneidad didáctica de planes formativos desarrollado por Godino (2013); Godino, Rivas y Arteaga (2012); Godino et al. (2013) quienes evalúan la idoneidad didáctica de procesos de enseñanza y aprendizaje de las matemáticas o estadística en profesores de primaria (niveles K-8). Por esta razón, en lo que sigue presentamos la versión revisada de la GVID-IDE.

\section{Resultados}

En esta sección sintetizamos el conjunto de indicadores de idoneidad organizados según las dos dimensiones descritas en la sección 2, la faceta epistémica y las demás facetas (cognitiva, afectiva, mediacional, instruccional y ecológica). Dicha colección de indicadores, se espera sea útil para el diseño y valoración de planes formativos dirigidos a futuros profesores de matemática en el área de didáctica de la estadística.

\subsection{Indicadores de idoneidad epistémica}

La primera dimensión, referida a la faceta epistémica, considera el conocimiento del contenido didáctico-estadístico a enseñar (desde el punto de vista institucional) que la comunidad de educadores estadísticos considera pertinentes para un aprendizaje idóneo u óptimo sobre didáctica de la estadística. De esta forma, según el enfoque teórico adoptado (GODINO et al., 2013) organizamos esta dimensión en las seis facetas de idoneidad didáctica, entendidas en cada caso como:

(1) Contenido Estadístico: referido a que el profesor comprenda la disciplina desde un punto de vista plural, identificando a la resolución de problemas como un medio para dar sentido al contenido, articulando lo funcional con lo lingüístico, procedimental y argumentativo. El Cuadro 4 presenta la colección de indicadores sobre este contenido. 


\section{Situaciones-Problema}

- Se incluyen problemas significativos del mundo real que puedan ser desarrollados por medio del proceso de resolución de problemas estadísticos (PPDAC), enfatizando en la omnipresente variabilidad.

- Se promueve el uso de problemas históricos que originaron el cálculo de probabilidades. Lenguajes

- Se incluyen distintas representaciones para explorar, resumir y describir patrones de variabilidad en datos unidimensionales categóricos y cuantitativos (tablas, gráficos, resúmenes numéricos).

- Se proponen diversas representaciones para describir patrones de asociación de dos variables, ya sean categóricas (tablas de doble entrada) o cuantitativas (gráfico de dispersión).

- Se propone el uso de tablas de doble entrada, diagramas de árbol y otras representaciones para la enseñanza de las probabilidades.

- Se promueve el uso del lenguaje escrito u oral para comunicar resultados de forma clara y precisa con el lenguaje estadístico.

\section{Reglas: Definiciones, proposiciones y procedimientos}

- Se promueve el trabajo con modelos estadísticos que describan la variabilidad de los datos (datos = estructura + variabilidad).

- Se propone transformar las preguntas de investigación en preguntas estadísticas (que puedan ser resueltas por medio de datos en el ciclo PPDAC).

- Se propone identificar y distinguir los distintos tipos de variables y datos al abordar una pregunta estadística.

- Se incluyen los conceptos, procedimientos y propiedades fundamentales de la estadística descriptiva.

- Se incluyen los principales conceptos, procedimientos y propiedades de las probabilidades y las variables aleatorias.

- Se promueven los conceptos y procedimientos primordiales para modelar la asociación entre variables por medio del análisis de regresión.

- Se promueven los principales conceptos, procedimientos, técnicas y propiedades de la inferencia estadística paramétrica.

- Se incluye diferenciar situaciones donde sea necesario un estudio observacional o un estudio comparativo. Argumentos

- Se promueve la construcción de argumentos viables, claros y precisos que comuniquen la utilidad y poder del pensamiento estadístico.

- Se incluye evaluar y criticar la plausibilidad de conclusiones alternativas.

- Se promueve la distinción entre el razonamiento estadístico correcto del defectuoso.

\section{Relaciones}

- Se promueve establecer conexiones entre el diseño del estudio y la interpretación de resultados.

- Se promueve interpretar los resultados de un estudio estadístico con aspectos como el sesgo y el alcance de inferencias.

- Se promueve relacionar la distribución de los datos y su variabilidad con modelos estadísticos sesgados y no sesgados.

- Se incluye conectar la simulación con procedimientos inferenciales.

- Se promueve valorar el contexto y la variabilidad en todo el proceso de resolución de problemas estadístico.

- Se promueve valorar el rol de los datos y la necesidad de producirlos considerando la variabilidad.

Cuadro 4 - Indicadores de idoneidad del Contenido Estadístico Fuente: Resultados de la investigación

(2) Contenido Cognitivo: referido al conocimiento especializado, por parte de los profesores, de la estadística en relación al aprendizaje de los estudiantes (considerando errores, obstáculos, dificultades recurrentes y adaptaciones curriculares a las diferencias individuales), la evaluación y sus conocimientos previos, cuyos indicadores se resumen en el Cuadro 5 . 


\section{Conocimientos Previos}

- Se propone trabajar desde el razonamiento informal para introducir la comprensión de tópicos de mayor dificultad para los estudiantes.

- Se promueve desarrollar no sólo el conocimiento del contenido disciplinar sino que también su progresión y conexión entre los distintos niveles escolares.

Adaptaciones curriculares según diferencias individuales

- Se incluye identificar estilos de aprendizaje, necesidades educativas especiales y talentos específicos de los estudiantes.

- Se promueve el uso pertinente de la evaluación diferenciada.

- Se promueve la inclusión de todos los estudiantes con sus respectivas diferencias sociales, sexuales, étnicas, de apariencia física y desarrollo académico.

\section{Aprendizaje}

- Se promueve estar familiarizados con las concepciones, dificultades y errores comunes de los estudiantes al aprender estadística, probabilidades e inferencia.

- Se incentiva centrar el foco de atención en los estudiantes del sistema escolar, sus características y modos de aprendizaje.

- Se promueve el uso de estrategias de enseñanza y evaluación para promover el aprendizaje.

\section{Cuadro 5 - Indicadores de idoneidad del Contenido Cognitivo}

Fuente: Resultados de la investigación

(3) Contenido Afectivo: abarca el conocimiento, comprensión y manejo de los intereses, necesidades, actitudes y emociones de los estudiantes en el aprendizaje de la estadística. Los indicadores inferidos para este contenido se presentan en el Cuadro 6.

Intereses y necesidades

- Se consideran los intereses y motivaciones de los estudiantes para en la propuesta de problemas y tareas estadísticas.

- Se promueven tareas y actividades que reconozcan la importancia de la disciplina en la sociedad.

- Se analizan situaciones reales para identificar modos de pensar, sentir y actuar de los estudiantes.

$$
\text { Actitudes }
$$

- Se promueve una actitud positiva hacia la resolución de problemas estadísticos.

- Se promueven actitudes como la perseverancia y crecimiento tanto intelectual como moral de los estudiantes.

\section{Emociones}

- Se promueve la seguridad física y afectiva de los estudiantes.

\section{Cuadro 6 - Indicadores de idoneidad del Contenido Afectivo}

Fuente: Resultados de la investigación

(4) Contenido Interaccional: referido a la capacidad del futuro profesor para comunicar adecuadamente el contenido estadístico, evaluar formativamente los aprendizajes e identificar y resolver conflictos relacionados con la manera de interactuar en el aula. Los indicadores de este contenido se resumen en el Cuadro 7.

\section{Interacción Docente-Estudiante}

- Se promueven estrategias que valoren el proceso de investigación estadística.

- Se promueve un estilo de instrucción interactivo, centrado en problemas reales y sensible al pensamiento del estudiante.

- Se promueve el logro de objetivos de aprendizaje por medio de tareas, recursos y evaluaciones efectivas y coherentes.

- Se incluye incentivar, acoger y potenciar la participación de los estudiantes en la instrucción.

Interacción entre Estudiantes

- Se promueven instancias para compartir y comunicar razonamientos y métodos entre estudiantes. Autonomía

- Se promueve el trabajo autónomo de los estudiantes en el proceso de resolución de problemas estadísticos. 
Evaluación Formativa

- La evaluación enfatiza en la comprensión en interpretación conceptual sobre lo procedimental y algorítmico.

- Se incluyen diversos métodos de evaluación que enfaticen en el proceso de resolución de problemas estadístico como preguntas de respuesta abierta o proyectos de investigación.

- Se integra la evaluación como parte del proceso de enseñanza y aprendizaje, retroalimenta y orienta estrategias para seguir progresando.

- Se promueve el uso sistémico y efectivo de la evaluación formativa.

Cuadro 7 - Indicadores de idoneidad del Contenido Interaccional Fuente: Resultados de la investigación

(5) Contenido Mediacional: referido al conocimiento de las posibilidades de uso y limitaciones de recursos manipulativos e informáticos (TIC's) en la enseñanza de la estadística y su gestión en el tiempo disponible, cuyos indicadores se resumen en el Cuadro 8.

\section{Recursos Materiales}

- Se promueve el uso de TIC's (calculadoras, hojas de cálculo, applets y dispositivos aleatorios) que potencien el desarrollo de la enseñanza de la estadística.

- Se promueve el uso de simulaciones computacionales y con material concreto para la enseñanza y aprendizaje de las probabilidades, variables aleatorias e inferencia estadística.

Número de estudiantes, horario y condiciones del aula

- Se promueve el uso adecuado del espacio, equipamiento y recursos del aula para potenciar el trabajo de los estudiantes.

- Se promueve generar en el aula y establecimiento educativo un espacio acogedor y estimulante para los estudiantes.

Tiempo para la enseñanza y el aprendizaje

- Se gestiona eficazmente el tiempo de la clase a favor del logro de los objetivos propuestos.

Cuadro 8 - Indicadores de idoneidad del Contenido Mediacional

Fuente: Resultados de la investigación

(6) Contenido Ecológico: referido al conocimiento del profesor respecto a las orientaciones curriculares y los factores externos (económicos, políticos, culturales) que intervienen en la enseñanza y aprendizaje de la estadística, junto a su capacidad de innovar basados en la investigación. Sus indicadores se presentan en el Cuadro 9.

\section{Adaptación del Currículo}

- Se conocen los enfoques pedagógicos y disciplinares del currículo y sus instrumentos para organizar la enseñanza.

- Se tiene en cuenta la normativa de integración de la discapacidad en el sistema escolar. Innovación didáctica

- Se promueve el uso de herramientas tecnológicas que potencien el proceso de enseñanza y aprendizaje. Adaptación socio-cultural y profesional

- Se promueve la generación de una cultura de respeto, seguridad y confianza en la escuela, que involucre a los estudiantes, sus familias y comunidades.

Educación en valores

- Se promueve la formación ciudadana de los estudiantes.

- Se incluye el desarrollo de valores, actitud crítica e inclusión en los estudiantes.

\section{Conexiones intra e interdisciplinares}

- Se promueve el trabajo colegiado entre diversos docentes o especialistas

- Se promueven oportunidades para integrar y potenciar los aprendizajes de las distintas áreas curriculares y sus interdependencias.

Cuadro 9 - Indicadores de idoneidad del Contenido Ecológico Fuente: Resultados de la investigación. 


\subsection{Otros indicadores de idoneidad}

Por otro lado, los procesos formativos de profesores deben tener en cuenta no sólo el contenido didáctico-estadístico a enseñar (faceta epistémica), sino que, además, las facetas restantes (cognitiva, afectiva, interaccional, mediacional y ecológica) implicadas en dicho proceso, que involucran al formador con los profesores en formación y conforman la segunda dimensión de la GVID-IDE que presentamos a continuación:

(1) Faceta Cognitiva: referida al logro efectivo de las expectativas de aprendizaje en didáctica de la estadística, donde el formador debe conocer las formas de razonar de sus estudiantes (futuros profesores), sus etapas de desarrollo cognitivo y estrategias, dificultades o posibles errores en el aprendizaje de la enseñanza de la estadística. Los indicadores resultantes para esta faceta se resumen en el Cuadro 10.

Conocimientos Previos

- Se promueve la comprensión de que los conceptos estadísticos se construyen se forma progresiva en la escuela.

\section{Aprendizaje}

- Se promueve experimentar el aprendizaje conceptual de las estadísticas a enseñar de forma similar a sus estudiantes.

- Se promueve el compromiso de los profesores en la resolución de problemas estadísticos.

- Se diferencia entre el pensamiento matemático del estadístico.

- Se promueve el correcto entendimiento de los modelos estadísticos, que se juzgan según su utilidad y descripción de los datos.

- Se promueve la comprensión del razonamiento estadístico desde la perspectiva de los datos y/o la simulación, destacando la variación presente y el contexto de cada situación.

- Se promueve el aprendizaje de la probabilidad, las variables aleatorias y sus componentes como base para identificar patrones de variabilidad que permitan cuantificar la incertidumbre.

- Se promueve el aprendizaje de la inferencia estadística, su finalidad, componentes y principales procedimientos de estimación.

- Se promueve la comprensión del razonamiento inferencial a través de la aleatorización y simulación.

- Se considera el aprendizaje del análisis de regresión lineal.

- Se incluye la preparación para conducir el aprendizaje de los tópicos de análisis de datos, probabilidades e inferencia estadística presentes en el currículo escolar.

Cuadro 10 - Indicadores de idoneidad de la Faceta Cognitiva

Fuente: Resultados de la investigación.

(2) Faceta Afectiva: abarca aspectos como las actitudes y motivaciones de los futuros profesores hacia la enseñanza de la estadística y hacia el contenido subyacente, que deben ser potenciadas positivamente por parte del formador. El Cuadro 11 contiene los indicadores que conforman esta faceta.

\section{Actitudes}

- Se fomenta una actitud sanamente escéptica frente a la información estadística presente en los medios.

- Se promueve una actitud de supervisión durante el proceso de resolución de problemas, evaluando continuamente la razonabilidad de los resultados.

- Se aprecia la eficacia del ciclo de investigación en la resolución de problemas.

\section{Cuadro 11 - Indicadores de idoneidad de la Faceta Afectiva}

Fuente: Resultados de la investigación. 
(3) Faceta Interaccional: referida a las competencias comunicativas del profesor en formación con su formador y pares, junto a su capacidad de trabajo autónomo, sumado al uso de la evaluación formativa por parte de los formadores. Los indicadores obtenidos para esta faceta se presentan en el Cuadro 12.

Interacción formador-futuro profesor
- Se incluye que los formadores alienten a sus estudiantes a esforzarse por mejorar su enseñanza
continuamente.
Autonomía del futuro profesor
- Se promueve la autonomía y perseverancia en la resolución de problemas a lo largo del ciclo de
investigación.
- Uso de la evaluación formativa
- Se potencia y valoriza la evaluación formativa.
- Se promueve que la evaluación enfatice en la comprensión e interpretación conceptual por sobre la
aplicación de fórmulas y algoritmos.
- Se fomenta que los formadores utilicen medios de evaluación apropiados a la disciplina, como los
proyectos estadísticos, que sean un ejemplo a sus prácticas futuras.

Cuadro 12 - Indicadores de idoneidad de la Faceta Interaccional

Fuente: Resultados de la investigación.

(4) Faceta Mediacional: referida al uso de recursos disponibles (informáticos, audiovisuales o de comunicación virtual) para el planteamiento de situaciones vinculadas con la práctica de la enseñanza y su análisis en los tiempos disponibles para ello. En este sentido, el Cuadro 13 contiene los indicadores de esta faceta.

\section{Recursos materiales}

- Se promueve el uso de herramientas tecnológicas para profundizar en la enseñanza de la disciplina y optimizar el tiempo destinado a ella.

Tiempo para la enseñanza y aprendizaje

- Se consideran al menos dos cursos destinados al estudio de la estadística y uno a su enseñanza.

Cuadro 13 - Indicadores de idoneidad de la Faceta Mediacional

Fuente: Resultados de la investigación.

(5) Faceta Ecológica: abarcar el conocimiento del formador en cuanto al currículo respectivo, el uso de nuevas tecnologías en la implementación de actividades formativas basadas en la investigación y la conexiones con otras áreas disciplinares, complementado con una formación en valores y pensamiento crítico. El Cuadro 14 resume los indicadores correspondientes a esta faceta.

Adaptación del currículo

- Se promueve la modelización de situaciones de la vida real.

- El currículo para futuros profesores incorpora aspectos del contenido estadístico y del conocimiento especializado para la enseñanza.

- Los cursos de profesores se alinean con los principales hallazgos de investigación y dan oportunidades idóneas de enseñanza. Innovación didáctica

- Se incluye el uso de softwares estadísticos que promuevan múltiples experiencias para analizar situaciones reales.

Adaptación socio-cultural y profesional

- Se promueve trabajar en formas propias de la disciplina para desenvolverse, de manera efectiva y eficiente, frente a las demandas profesionales que le impone la sociedad. 


\section{Educación en valores}

- Se potencia una sólida formación en valores como la responsabilidad, perseverancia y comportamiento ético.

\section{Conexiones intra e interdisciplinares}

- Se fomenta la aplicación de las matemáticas para ayudar a responder a preguntas estadísticas que surgen en la vida diaria.

- Se promueve el reconocimiento de las conexiones verticales de los temas estadísticos entre los niveles educativos.

- Se promueve el reconocimiento de las conexiones horizontales con el currículo de matemáticas y otras áreas temáticas.

Cuadro 14 - Indicadores de idoneidad de la Faceta Ecológica

Fuente: Resultados de la investigación.

\section{Reflexiones finales}

En este trabajo hemos abordado el problema de la valoración de planes de formación de profesores de matemática en didáctica de la estadística a través de la construcción de una Guía de Valoración de Idoneidad Didáctica de procesos de Instrucción en Didáctica de la Estadística (GVID-IDE), que sintetiza los principios curriculares que orientan dicho proceso, presentes en directrices de consenso en la comunidad educativa y elaboradas a partir de los principales resultados de investigaciones en este campo. En este punto, es importante destacar que si bien los documentos consultados son específicos para Estados Unidos y Chile, pensamos que con el desglose presentado a lo largo de este artículo permitirá a otros investigadores consultar sus directrices y complementarlas con estos resultados.

De esta forma, podemos posicionar la GVID-IDE dentro de la familia de instrumentos de esta índole (p. e.: GODINO, 2013; GODINO et al., 2013; GODINO; RIVAS; ARTEAGA, 2012) cuyo manejo, como mencionamos en la sección 2.1, depende del actor educativo que la utilice. Si la aplicamos al profesor (o futuro profesor), la faceta epistémica abarca el conocimiento especializado del contenido respecto a sus estudiantes y en el caso del formador de profesores, las demás facetas le permitirán orientar, adaptar y desarrollar procesos de estudio de didáctica de la estadística.

Más aún, en el marco del Modelo de Conocimientos y Competencias del profesor de matemáticas - CCDM (GODINO et al., 2017, p. 12), el futuro profesor de matemáticas (o formador) debe "adquirir competencia de análisis de la idoneidad didáctica de los procesos de estudio", de manera de utilizar este recurso para la reflexión global sobre la práctica didáctica, su valoración y mejora paulatina. Por esta razón, la construcción de la GVID-IDE y su posterior implementación puede considerarse un ejemplo de dicha competencia, que aporta con el proceso de reflexión meta-didáctica del proceso de formación de profesores en didáctica de la estadística. 
Esperamos que esta guía sea un insumo valioso tanto para formadores de profesores como para quienes tengan la responsabilidad de diseñar o evaluar planes formativos para futuros docentes en el campo de educación estadística.

\section{Referencias}

BALL, D. Working on the inside: Using one's own practice as a site for stuying teaching and learning. En: KELLY, A. Y LESH, R. (Ed.). Handbook of Research Design Mathematics and Science Education. London: Lawrence Erlbaum, 2000. p. 365-402.

BATANERO, C.; BURRILL, G.; READING, C. Challenges for Teaching Statistics in School Mathematics, and Preparing Mathematics Teachers. En: BATANERO, C.; BURRIL, G.; READING, C. (Ed.). Teaching statistics in school mathematics: Challenges for teaching and teacher education. New York: Springer, 2011. p. 407-418.

BELTRÁN-PELLICER, P.; GODINO, J. Aplicación de indicadores de idoneidad afectiva en un proceso de enseñanza de probabilidad en educación secundaria. Perspectiva Educacional Formación de Profesores, Valparaíso, v. 56, n. 2, p. 92-116, 2017.

BEN-ZVI, D.; MAKAR, K. International Perspectives on the Teaching and Learning of Statistics. En: BEN-ZVI, D.; MAKAR, K. (Ed.). The Teaching and Learning of Statistics. Suiza: Springer, 2016. p. 1-19.

BREDA, A.; FONT, V.; LIMA, V. M. R. A noção de idoneidade didática e seu uso na formação de professores de matemática. Jornal Internacional de Estudos em Educação Matemática, São Paulo, v. 8, n. 2, p. 1-41, 2015.

FRANKLIN, C. et al. Guidelines for Assessment and Instruction in Statistics Education (GAISE) Report. 1. ed. Alexandria: American Statistical Association, 2007. Disponible en: <http://www .amstat.org/education/gaise/GAISEPreK-12_Full.pdf>. Acceso en: 9 abril 2017.

FRANKLIN, C. et al. Statistical Education of Teachers (SET). 1. ed. Alexandria: American Statistical Association, 2015. Disponible en: <www.amstat.org/education/SET/SET.pdf>. Acceso en: 5 mayo 2017.

GAISE COLLEGE REPORT ASA REVISION COMMITTE. Guidelines for Assessment and Instruction in Statistics Education (GAISE) College Report. 2. ed. Alexandria: American Statistical Association, 2016. Disponible en:

<http://www.amstat.org/asa/files/pdfs/GAISE/GaiseCollege_Full.pdf>. Acceso en: 9 abril 2017.

GAL, I. Adult's Statistical Literacy: Meanings, Components, Responsibilities. International Statistical Review, The Netherlands, v. 70, n. 1, p. 1-51, 2002.

GODINO, J.; CONTRERAS, A.; FONT, V. Análisis de procesos de instrucción basado en el enfoque ontológico-semiótico de la cognición matemática. Recherches en Didactique des Mathématiques, Grenoble, Franca, v. 26, n. 1, p. 39-88, 2006.

GODINO, J. et al. Análisis y valoración de la idoneidad didáctica de procesos de estudio de las matemáticas. Paradigma, Maracay, v. 27, n. 2, p. 221-252, 2006.

GODINO, J.; BATANERO, C.; FONT, V. The onto-semiotic approach to research in mathematics education. ZDM. The International Journal on Mathematics Education, Berlín, v. 39, n. 1-2, p. 127$135,2007$. 
GODINO, J. Categorías de Análisis de los conocimientos del profesor de matemáticas. Revista UNIÓN, San Cristóbal de La Laguna, n. 20, p. 13-31, 2009.

GODINO, J. et al. Models for statistical pedagogical knowledge. En: BATANERO, C.; BURRIL, G.; READING, C. (Ed.). Teaching statistics in school mathematics: Challenges for teaching and teacher education. New York: Springer, 2011. p. 271-282.

GODINO, J. Origen y aportaciones de la perspectiva ontosemiótica de investigación desde la Didáctica de la Matemática como disciplina científica. En: ESTEPA, A. et al. (Ed.). Investigación en Educación Matemática XVI. Jaén: SEIEM, 2012. p. 49-68.

GODINO, J.; RIVAS, H.; ARTEAGA, P. Inferencia de indicadores de idoneidad didáctica a partir de orientaciones curriculares. Práxis Educativa, Ponta Grossa, v. 7, n. 2, p. 331-354, 2012.

GODINO, J. Indicadores de la Idoneidad didáctica de procesos de enseñanza y aprendizaje de las matemáticas. Cuadernos de Investigación y Formación en Educación Matemática, Costa Rica, v. 8, n. 11, p. 111-132, 2013.

GODINO, J. et al. Componentes e indicadores de idoneidad de programas de formación de profesores en didáctica de la matemática. REVEMAT, Florianópolis, v. 8, n. 1, p. 46-74, 2013.

GODINO, J. et al. Enfoque Ontosemiótico de los Conocimientos y Competencias del profesor de matemáticas. Bolema, Rio Claro (SP), v. 31, n. 57, p. 90-113, 2017.

HERNÁNDEZ, R.; FERNÁNDEZ, C.; BAPTISTA, P. Metodología de la Investigación. 6. ed. México: editorial McGraw Hill Education, 2014.

HILL, H.; BALL, D.; SCHILLING, S. Unpacking pedagogical content knowledge: Conceptualizing and measuring teachers' topic-specific knowledge of students. Journal for Research in Mathematics Education, Reston, V.A., v. 39, n. 4, p. 372-400, 2008

KRIPPENDORFF, K. Metodología de análisis de contenido: teoría y práctica. México: Paidós, 1990.

MINISTERIO DE EDUCACIÓN (MINEDUC). CURRICULUM: Objetivos Fundamentales y Contenidos Mínimos Obligatorios de la Educación Básica y Media. Gobierno de Chile, Santiago, diciembre 2009, p. 145-194.

MINISTERIO DE EDUCACIÓN (MINEDUC); CENTRO DE PERFECCIONAMIENTO, EXPERIMENTACIÓN E INVESTIGACIONES PEDAGÓGICAS (CPEIP). Estándares orientadores para Carreras de pedagogía en educación media. 2012. Disponible en: <http://p ortales.mineduc.cl/usuarios/cpeip/File/librosestandaresvale/libromediafinal.pdf >. Acceso en: 5 mayo 2017.

MINISTERIO DE EDUCACIÓN, CULTURA Y DEPORTE (MECD). Boletín oficial del estado. Orden ECD/65/2015, de 21 de enero, por la que se describen las relaciones entre las competencias, los contenidos, los criterios de evaluación de la educación primaria, secundaria y el bachillerato. Gobierno de España, n. 25, enero 2015. p. 6986-7003.

NATIONAL COUNCIL OF TEACHERS OF MATHEMATICS (NCTM). Principles and standards for school mathematics. 2000. Disponible en: 〈http://www.nctm.org/standards >. Acceso en: 6 junio 2017.

NATIONAL GOVERNORS ASSOCIATION CENTER FOR BEST PRACTICES; COUNCIL OF 
CHIEF STATE SCHOOL OFFICERS. Common Core State Standards for Mathematics, 2011. Disponible en: <http://www.corestandards.org/assets/CCSSI_Math\%20Stan dards.pdf>. Acceso en: 6 junio 2017.

PINO-FAN, L.; GODINO, J. Perspectiva ampliada del conocimiento didáctico-matemático del profesor. Paradigma, Maracay, v. 36, n. 1, p. 87-109, 2015.

SCHOENFELD, A.; KILPATRICK, J. Toward a theory of proficiency in teaching mathematics. En: TIROSH, D.; WOOD, T. (Ed.). Tools and processes in mathematics teacher education. Rotterdam: Sense Publishers, 2008. p. 321-354.

SHULMAN, L. Knowledge and teaching: Foundations of the new reform. Harvard Educational Review, Massachusetts, v. 57, n. 1, p. 1-22, 1987.

Submetido em 19 de Janeiro de 2018. Aprovado em 31 de Julho de 2018. 